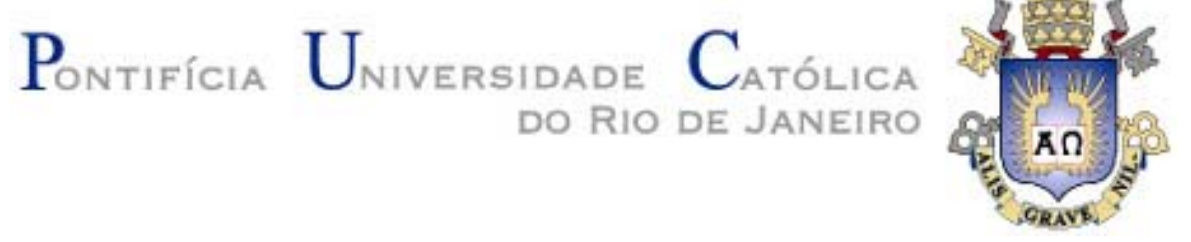

Thiago Cardoso Teixeira

Comparando Black-Scholes e Corrado-Su: um estudo sobre a volatilidade implícita aplicado ao mercado brasileiro de opções de compra de ações

Dissertação de Mestrado

Dissertação apresentada ao Programa de PósGraduação em Administração de Empresas da PUCRio como requisito parcial para obtenção do título de Mestre em Administração de Empresas

Orientador: Prof. Antonio Carlos Figueiredo Pinto

Rio de Janeiro

Agosto de 2011 
Thiago Cardoso Teixeira

\section{Comparando Black-Scholes e Corrado-Su: um estudo sobre a volatilidade implícita aplicado ao mercado brasileiro de opções de compra de ações}

Dissertação apresentada como requisito parcial para obtenção do grau de Mestre pelo Programa de Pósgraduação em Administração de Empresas da PUC-Rio. Aprovada pela Comissão Examinadora abaixo assinada.

Prof. Antonio Carlos Figueiredo Pinto

Orientador

Departamento de Administração - PUC-Rio

Prof. Marcelo Cabus Klotzle Departamento de Administração - PUC-Rio

Prof. Marco Antonio Cunha de Oliveira FACC - UFRJ

Prof ${ }^{a}$. Mônica Herz

Vice-Decana de Pós-Graduação do CCS

Rio de Janeiro, 26 de agosto de 2011 
Todos os direitos reservados. É proibida a reprodução total ou parcial do trabalho sem autorização da universidade, do autor e do orientador.

Thiago Cardoso Teixeira

Graduou-se em Ciências Econômicas pela Escola Brasileira de Economia e Finanças da Fundação Getulio Vargas em 2007

Ficha Catalográfica

Teixeira, Thiago Cardoso

Comparando Black-Scholes e Corrado-Su: um estudo sobre a volatilidade implícita aplicado ao mercado brasileiro de opções de compra de ações I Thiago Cardoso Teixeira ; orientador: Antonio Carlos Figueiredo Pinto. - 2011.

60 f. : il. (color.) ; $30 \mathrm{~cm}$

Dissertação (mestrado)-Pontifícia Universidade Católica do Rio de Janeiro, Departamento de Administração, 2011. Inclui bibliografia

1. Administração - Teses. 2. Volatilidade realizada. 3. Volatilidade implícita. 4. Opções de compra. I. Pinto, Antonio Carlos Figueiredo. II. Pontifícia Universidade Católica do Rio de Janeiro. Departamento de Administração. III. Título. 


\section{Agradecimentos}

Gostaria de agradecer, em primeiro lugar, ao grande amigo Jose Carlos Nogueira Cavalcante Filho por todo auxílio com a parte técnica e quantitativa deste estudo.

Ao Professor Antonio Carlos Figueiredo (PUC) por toda atenção e conselhos desde o início.

Ao Professor Edson Daniel Lopes Gonçalves (FGV) por todas as idéias no decorrer do trabalho. 


\section{Resumo}

Teixeira, Thiago Cardoso; Pinto, Antonio Carlos Figueiredo. Comparando Black-Scholes e Corrado-Su: um estudo sobre a volatilidade implícita aplicado ao mercado brasileiro de opções de compra de ações. Rio de Janeiro, 2011. 60p. Dissertação de Mestrado - Departamento de Administração, Pontifícia Universidade Católica do Rio de Janeiro.

Algumas literaturas sugerem que a volatilidade implícita das opções de compra de ações não deve ser utilizada como estimador para a volatilidade futura. Contudo, estudos recentes e aplicados ao mercado brasileiro de ações comprovaram que em determinados casos existe relação entre a volatilidade implícita e a volatilidade real (ou realizada). Isso significa dizer que a primeira traz informações sobre a última. Nesse contexto, o objetivo deste estudo é comparar a volatilidade implícita de dois modelos de apreçamento de opções com a volatilidade realizada. Entre os modelos de Black-Scholes (1973) e Corrado-Su (1996), utilizando dados de opções de Petrobras e Vale do Rio Doce, foram calculados, através do erro quadrático, aqueles resultados que mais se aproximaram da volatilidade realizada. Estes resultados trazem indícios de que o modelo de Black-Scholes, em média, foi superior ao Corrado-Su no período que vai de janeiro de 2005 a julho de 2009. Porém, o último, por levar em consideração a assimetria e a curtose da distribuição de retornos, chegou mais perto da volatilidade realizada apenas em alguns momentos específicos das economias brasileira e mundial.

\section{Paravras-chave}

Volatilidade realizada; volatilidade implícita; opções de compra 


\section{Abstract}

Teixeira, Thiago Cardoso; Pinto, Antonio Carlos Figueiredo (Advisor). Comparing Black-Scholes and Corrado-Su: a study on implied volatility applied to the brazilian call option market. Rio de Janeiro, 2011. 60p. MSc. Dissertation - Departamento de Administração, Pontifícia Universidade Católica do Rio de Janeiro.

Several authors have proposed that implied volatility from purchase options should not be used as an estimate for future volatility. However, recent studies applied to the Brazilian stock market proved that in certain cases there is relation between implied volatility and realized volatility. This means that the first one provides information on the last. In this context, the objective of this study is to compare implied volatilities from two different option pricing models against the realized volatility. The models are Black-Scholes (1973) and Corrado-Su (1996). Working with purchase options on Petrobras and Vale do Rio Doce, it was calculated the difference, by quadratic error, between the implied volatility of these models and the realized volatility. After this, it was checked those results that came closer to the realized volatility. The results provide evidence that the Black-Scholes model, on average, has higher performance than Corrado-Su from January 2005 to July 2009. However, Corrado-Su by taking into account the asymmetry and kurtosis of the distribution of returns came closer to the realized volatility only in specific moments of the Brazilian and global economies.

\section{Keywords}

Realized volatility; implied volatility; purchase options. 


\section{Sumário}

1. Introdução 11

2. Referencial Teórico 13

2.1. Conceitos Básicos sobre Opções 13

2.2. O Modelo de Black e Scholes 14

2.3. O Modelo Corrado-Su e a Restrição de Martingale 16

2.4. A Volatilidade Implícita 20

3. Metodologia e Base de Dados 31

3.1. Base de Dados $\quad 31$

3.2. Metodologia 33

4. Resultados 36

4.1. Vale $\quad 37$

4.2. Petrobrás 39

4.3. Vale e Petrobrás: Resultados Utilizando Janelas de
Assimetria e Curtose Históricas de 44 dias Úteis

4.3.1. Vale $\quad 41$

4.3.2. Petrobrás 43

5. Conclusão 45

6. Códigos VBA para Algoritmo Utilizado no Cálculo da

Volatilidade Implícita do Modelo Corrado-Su (1996) 46

7. Referências Bibliográficas $\quad 57$ 


\section{Lista de figuras}

Figura 1 - Probabilidade cumulativa de uma variável padronizada

Figura 2 - Evolução do retorno com base 100 para as duas ações no período analisado 


\section{Lista de gráficos}

Gráfico 1 - Volatility Smiles 27

Gráfico 2 - Volatilidade realizada mensal anualizada no período analisado

Gráfico 3 - Janela móvel de volatilidade realizada anualizada para 21 dias de 03/01/2005 a 31/07/2009

Gráfico 4 - Corrado-Su (1996) e Black-Scholes (1973): percentual de menos distância em relação a volatilidade realizada

Gráfico 5 - Percentual em que Corrado-Su (1996) mais se aproximou da volatilidade realizada para Vale

Gráfico 6 - Percentual em que Corrado-Su (1996) mais se aproximou da volatilidade realizada para Petrobrás

Gráfico 7 - Percentual em que Corrado-Su (1996) mais se aproximou da volatilidade realizada utilizando uma janela de 44 dias úteis de assimetria e curtose históricas e o critério de moneyness.

Gráfico 8 - Percentual em que Corrado-Su (1996) mais se aproximou da volatilidade realizada utilizando uma janela de 44 dias úteis de assimetria e curtose históricas e o critério de moneyness para Vale

Gráfico 9 - Percentual em que Corrado-Su (1996) mais se aproximou da volatilidade realizada utilizando uma janela de 44 dias úteis de assimetria e curtose históricas e o critério de moneyness para Petrobras 


\section{Lista de tabelas}

Tabela 1 - Estatísticas descritivas de Vale (22 dias úteis)

Tabela 2 - Estatísticas descritivas de Petrobrás (22 dias úteis)

Tabela 3 - Estatísticas descritivas de Vale (44 dias úteis)

Tabela 4 - Estatísticas descritivas de Petrobras (44 dias úteis) 